\title{
Rice Root Genetic Architecture: Meta-analysis from a Drought QTL Database
}

\author{
Brigitte Courtois • Nourollah Ahmadi • Farkhanda Khowaja • Adam H. Price • \\ Jean-François Rami • Julien Frouin • Chantal Hamelin • Manuel Ruiz
}

Received: 7 April 2009 /Accepted: 30 June 2009/Published online: 26 August 2009

(C) The Author(s) 2009. This article is published with open access at Springerlink.com

\begin{abstract}
During the last 10 years, a large number of quantitative trait loci (QTLs) controlling rice root morphological parameters have been detected in several mapping populations by teams interested in improving drought resistance in rice. Compiling these data could be extremely helpful in identifying candidate genes by positioning consensus QTLs with more precision through meta-QTL analysis. We extracted information from 24 published papers on QTLs controlling 29 root parameters including root number, maximum root length, root thickness, root/ shoot ratio, and root penetration index. A web-accessible database of 675 root QTLs detected in 12 populations was constructed. This database includes also all QTLs for drought resistance traits in rice published between 1995 and 2007. The physical position on the pseudochromosomes of the markers flanking each QTL was determined. An overview of the number of root QTLs in $5-\mathrm{Mb}$ segments covering the whole genome revealed the existence of "hot spots," The 32 trait $\times$ chromosome combinations comprising six or more QTLs were subjected to a meta-QTL analysis using the software package MetaQTL. The method enabled us both to determine the likely number of true QTLs in these areas using an Akaike
\end{abstract}

Electronic supplementary material The online version of this article (doi:10.1007/s12284-009-9028-9) contains supplementary material, which is available to authorized users.

B. Courtois $(\square) \cdot$ N. Ahmadi $\cdot$ J.-F. Rami $\cdot$ J. Frouin $\cdot$

C. Hamelin · M. Ruiz

UMR DAP, Cirad,

TA A96/03, Avenue Agropolis,

34398 Montpellier Cedex 5, France

e-mail: brigitte.courtois@cirad.fr

F. Khowaja $\cdot$ A. H. Price

University of Aberdeen,

Aberdeen AB24 3UU, UK information criterion and to estimate their position. The meta-QTL confidence intervals were notably reduced and, for the smallest ones, encompassed only a few genes.

Keywords Rice $\cdot$ Drought avoidance $\cdot$ Root morphology . QTLs $\cdot$ Meta-analysis

\section{Introduction}

Rice (Oryza sativa L.) is the main food crop and source of calorie intake for one third of the world's population. Although rice is often considered as an aquatic crop, more than $45 \%$ of the rice-growing area is not permanently flooded (International Rice Research Institute 2002). Drought is a major factor involved in yield instability in both the upland and rainfed lowland ecosystems (Widawski and O'Toole 1990). Drought resistance is therefore an important selection criterion in such ecosystems. Many traits are said to contribute to drought resistance (Ludlow and Muchow 1990; Fukai and Cooper 1995), but none is effective in all agronomic situations. Avoidance strategies that enable a plant to maintain its water status during water stress appear to be a reasonable option in a broad range of agronomic situations. A deep root system able to extract water at depth and respond to evaporative demand, provided there is water in the profile, is the most consensual of the traits contributing to drought avoidance at least in upland conditions (Yoshida and Hasegawa 1982; Nguyen et al. 1997; Price et al. 2002). In addition to its role in buffering drought effects, the root system plays an important role in anchoring the plant, capturing mineral resources, and in early vigor and competitiveness with weeds. It is also the site of major hormone biosynthesis. 
The development of the root system is controlled by constitutive intrinsic pathways that determine its global architecture as a function of the genetic potential of the accessions for root development and by response pathways that modulate its development in response to stress (water stress, submergence, nutrient deficiencies, etc.) and contribute to root developmental plasticity (Malamy 2005).

Descriptors of root development generally include maximum root length (MRL), which determines the baseline for access to nutrient and water resources, and root number (RN), root thickness (RTHK), and root length density, which determine the intensity of colonization of the soil profile and the ability to exploit soil resources within the baseline. Root length density is very strongly correlated with root dry weight (RDW), a parameter that is easier to measure (Yadav et al. 1997). Root dry weight has frequently been subdivided according to the layers where the roots were collected, with special attention paid to the deepest roots through parameters such as deep root weight (DRW) or the deep root weight to total root weight ratio (DRR). The thickness of the roots is strongly correlated with the diameter of the xylem vessels, and thick roots contribute to better water flux with less risk of cavitation (Yambao et al. 1992). In rice, root emission is synchronized with tiller emission following the phyllochron model (Nemoto et al. 1995). Allometric ratios such as rootto-shoot weight ratio $(\mathrm{R} / \mathrm{S})$, root dry weight per tiller, $(\mathrm{R} / \mathrm{T})$, deep root to shoot weight (DR/S), or deep root weight per tiller (DR/T) are used to describe the coordination between growth and development of the roots and shoots. A more precise description of the morphology and anatomy of the rice root system at tissue level has recently been provided (Rebouillat et al. 2008).

In $O$. sativa, significant genetic variation has been observed in root number, diameter, depth, branching, vertical density distribution, root-to-shoot ratio, water extraction, and root penetration (O'Toole and Bland 1987; Lafitte et al. 2001). The pattern of variation reflects the organization of $O$. sativa in six isozymic groups as described by Glaszmann (1987). The major differentiation is between isozymic group 1 (indicas), characterized by a superficial, thin, highly branched root system with a low $\mathrm{R} / \mathrm{S}$ ratio, and isozymic group 6 (japonicas) characterized by a deep, thick, less branched root system with a high R/S ratio. Most of the diversity is distributed between groups rather than within groups, with the exception of isozymic groups 2 and 6 which have a substructure linked to adaptation to the ecosystem, e.g., temperate irrigated versus tropical upland accessions in group 6 and irrigated boro versus upland aus accessions in group 2 (Courtois et al. 1996; Lafitte et al. 2001).

Following the development of molecular genetic approaches, the genetic architecture of the rice root system has been extensively studied by teams interested in improving drought resistance in rice. At least 24 papers have been published in the last 10 years on quantitative trait locus (QTL) detection for a large number of root parameters in several populations and different growth conditions (Champoux et al. 1995; Ray et al. 1996; Redona and Mackill 1996; Yadav et al. 1997; Price et al. 1999, 2000, 2002; Ali et al. 2000; Hemamalini et al. 2000; Tripathy et al. 2000; Zheng et al. 2000, 2003; Shen et al. 2001; Zhang et al. 2001a, b; Kamoshita et al. 2002a, b; Courtois et al. 2003; Nguyen et al. 2004; Xu et al. 2004; Li et al. 2005b; Horii et al. 2006; Yue et al. 2006; MacMillan et al. 2006).

These studies led to marker-aided selection, enabling validation of the position and effect of some of the QTLs (Shen et al. 2001; Steele et al. 2006) and to on-going QTL cloning work (Ahmadi et al., unpublished results).

Most papers mentioned above included a comparison of QTL positions across studies. Such comparisons are informative, but tedious to undertake at a large scale. To enable an easier shift from QTLs to the underlying candidate genes, progress is needed in combining the large body of QTL information. There are several possible ways to combine data from different studies to detect consensus QTLs and narrow QTL confidence intervals. One way would be to reanalyze the initial datasets in a combined way as was done in mouse by assuming a common biallelic mode of inheritance in several mapping populations ( $\mathrm{Li}$ et al. 2005a). The rice mapping populations have characteristics that would fit such a design well. Most populations are recombinant inbred lines (RIL) or doubled haploid $(\mathrm{DH})$ indica $\times$ japonica populations where two major alleles segregate, and they share a large corpus of markers, notably restriction fragment length polymorphism and microsatellite markers from Cornell University or from the Japanese Rice Genome Project (Harushima et al. 1998). However, this approach is limited by the difficulty involved in accessing raw phenotypic data. Another option is to perform meta-QTL analysis using the method proposed by Goffinet and Gerber (2000) and improved by Veyrieras et al. (2007). This statistical approach enables QTL results from independent studies to be combined into a single result using only published QTL data. Software packages based on this methodology are available such as BioMercator (Arcade et al. 2004) and Meta-QTL (Veyrieras et al. 2007), a new software that removes some of the limitations of BioMercator in the number of QTLs it can handle at once. Meta-QTL analyses for flowering time in maize (Chardon et al. 2004), resistance to a nematode in soybean (Guo et al. 2006), earliness in bread wheat (Hanocq et al. 2007), and $\mathrm{N}$ uptake in maize (Coque et al. 2008) have already shown the usefulness of this approach in determining the number of "true" QTLs underlying the observed QTLs and in positioning consensus QTLs with more 
precision. In rice, Norton et al. (2008) conducted a study on root QTLs, but it was limited to the Azucena $\times$ Bala population and only three QTL clusters.

The main objective of this study was therefore to combine the results of all QTL detection studies conducted from 1995 to 2007 on root traits in rice through a metaQTL analysis involving the whole genome.

Such an approach requires easy access to the entire QTL dataset for the traits and species under study. Many reported rice QTLs have already been assembled in the Gramene database (http://www.gramene.org) which is the broadest and so far most extensive source of public information on QTLs in cereals. However, in Gramene, specific information on the QTLs includes flanking markers, logarithm of the odds (LOD) score and phenotypic variation explained by the QTLs, but not the experimental conditions under which the QTLs were detected, which can be important when dealing with abiotic stresses. Information such as the mapping population size, which can be needed to compute the QTL confidence interval (Darvasi and Soller 1997), is not directly linked to the QTL. The link between the QTLs and the genome sequence is also indirect. The secondary objective of this study was therefore to develop a database on rice QTLs for drought resistance from which it will be easy to extract the elements necessary for meta-QTL analyses on drought-related traits.

\section{Results}

Database development

We extracted the relevant information on 1,467 QTLs controlling drought traits in rice from 42 published papers. An additional 23 papers were also analyzed, bringing the total number of QTLs in the database to 2,137, but their topic (QTLs for flowering time, QTL for tolerance to salinity, and QTLs detected in the IR64xAzucena population) was not relevant for the present paper. Basic information on QTLs is available to the scientific community in the rice module of the Tropgene database (http:// tropgenedb.cirad.fr/en/rice.html), which is devoted to genetic, genomic, and phenotypic information on tropical crops (Ruiz et al. 2004). In comparison with the original papers, the names of the traits have sometimes been modified for the purpose of homogenization. A definition of the traits largely inspired by Gramene and connected to the Trait Ontology was established and is available in Tropgene DB (http://tropgenedb.cirad.fr/html/rice_QTL.html).

The list of parameters recorded for each QTL is given in Supplementary Table 1. The list includes the "experimental conditions" [field or greenhouse conditions, aerobic or anaerobic conditions, and controlled (well watered, normal impedance) or stressed conditions (water stress or compaction)], which are particularly important for QTLs involved in response to abiotic stresses. The QTL set can therefore be split according to this parameter and meta-QTL analyses run on a specific subset of conditions.

The parameter list also includes the QTL positions with reference to the sequence of the Nipponbare pseudochromosomes v5.0 to facilitate the shift from the QTL and meta-QTL confidence intervals to the underlying genes. The positions of the QTLs were determined by the physical position of the most significant markers for QTLs mapped through single marker analysis. For QTLs detected through interval or composite interval mapping, their positions were defined as the mid-values of the physical positions of the two markers flanking the QTL peak because the names of flanking markers were always indicated in the papers, while this was seldom the case for estimated QTL positions. To determine the physical positions of the markers, Gramene data or BLAST results based on Gramene sequence information were used when available. Otherwise, for example in the case of amplified fragment length polymorphisms (AFLPs), the nearest marker with a known sequence in the original genetic map was used as a proxy. This solution led to a loss of precision in positioning the marker, but in our dataset, AFLPs represented only $5.9 \%$ of the markers linked to QTLs. Information on the physical position of each marker or of the proxy we used is also available in Tropgene DB (http://tropgenedb.cirad.fr/html/rice_Marker.html). For original data or additional details on a particular experiment, the reader is referred to the initial publications in the list of references. These QTLs can be visualized on a synthetic map using the Comparative Map Viewer Cmap v 0.13 from the GMOD project (http://www.gmod.org) integrated into Tropgene DB (http://tropgenedb.cirad.fr/en/ rice.html).

\section{Root QTLs}

From our QTL database, we extracted all the QTLs related to root traits. They come from the 24 papers listed in Table 1. For the Bala $\times$ Azucena population, a new more saturated genetic map was recently developed (Khowaja et al. 2009) and used to reanalyze the initial phenotypic data (Price et al. 1999, 2000, 2002; MacMillan et al. 2006), so the new QTLs were used in this study. The list of the traits with their acronym is given in Table 2. A few QTLs with a LOD score below 2.0 were discarded from the analysis to reduce the risk of including false positives.

The root QTL set was composed of 675 QTLs for 29 root traits detected in 12 mapping populations (Tables 1 and 2). In several cases, the same populations had been used by different teams in independent experiments. These 
Table 1 Rice Root QTL

Studies Included in the Analysis

\begin{tabular}{|c|c|c|c|c|}
\hline Ref. no & Reference & Population & Cross type & Pop. type \\
\hline 1 & Ali et al. (2000) & IR58821/IR52561 & RILs F7 & $\mathrm{I} / \mathrm{I}$ \\
\hline 2 & Champoux et al. (1995) & Co39/Moroberekan & RILs F7 & $\mathrm{I} / \mathrm{TJ}$ \\
\hline 3 & Courtois et al. (2003) & IAC $165 / \operatorname{Co} 39$ & RILs F7 & $\mathrm{TJ} / \mathrm{I}$ \\
\hline 4 & Hemamalini et al. (2000) & IR64/Azucena & $\mathrm{DH}$ & $\mathrm{I} / \mathrm{TJ}$ \\
\hline 5 & Horii et al. (2006) & Akihikari/IRAT109 & $\mathrm{BC} 1 \mathrm{~F} 4$ & $\mathrm{tJ} / \mathrm{TJ}$ \\
\hline 6 & Kamoshita et al. (2002a) & CT9993/IR62266 & $\mathrm{DH}$ & $\mathrm{TJ} / \mathrm{I}$ \\
\hline 7 & Kamoshita et al. (2002b) & CT9993/IR62266 & $\mathrm{DH}$ & $\mathrm{TJ} / \mathrm{I}$ \\
\hline 8 & Li et al. (2005b) & IRAT109/Yuefu & $\mathrm{DH}$ & $\mathrm{TJ} / \mathrm{tJ}$ \\
\hline 9 & MacMillan et al. (2006) & Bala/Azucena & RILs F6 & $\mathrm{I} / \mathrm{TJ}$ \\
\hline 10 & Nguyen et al. (2004) & CT9993/IR62266 & $\mathrm{DH}$ & $\mathrm{TJ} / \mathrm{I}$ \\
\hline 11 & Price et al. (1999) & Bala/Azucena & RILs F6 & $\mathrm{I} / \mathrm{TJ}$ \\
\hline 12 & Price et al. (2000) & Bala/Azucena & RILs F6 & $\mathrm{I} / \mathrm{TJ}$ \\
\hline 13 & Price et al. (2002) & Bala/Azucena & RILs F6 & $\mathrm{I} / \mathrm{TJ}$ \\
\hline 14 & Ray et al. (1996) & Co39/Moroberekan & RILs F7 & $\mathrm{I} / \mathrm{TJ}$ \\
\hline 15 & Redona and Mackill (1996) & Labelle/Black Gora & $\mathrm{F} 2: 3$ & $\mathrm{tJ} / \mathrm{A}$ \\
\hline 16 & Shen et al. (2001) & IR64/Azucena & $\mathrm{DH}$ & $\mathrm{I} / \mathrm{TJ}$ \\
\hline 17 & Tripathy et al. (2000) & CT9993/IR62266 & $\mathrm{DH}$ & $\mathrm{TJ} / \mathrm{I}$ \\
\hline 18 & Xu et al. (2004) & Zhenshan97/Minghui63 & RILs F10 & $\mathrm{I} / \mathrm{I}$ \\
\hline 19 & Yadav et al. (1997) & IR64/Azucena & $\mathrm{DH}$ & $\mathrm{I} / \mathrm{TJ}$ \\
\hline 20 & Yue et al. (2006) & Zhenshan97/IRAT109 & RILs F9 & $\mathrm{I} / \mathrm{TJ}$ \\
\hline 21 & Zhang et al. (2001a) & CT9993/IR62266 & $\mathrm{DH}$ & $\mathrm{TJ} / \mathrm{I}$ \\
\hline 22 & Zhang et al. (2001b) & IR1552/Azucena & RILs F10 & $\mathrm{I} / \mathrm{TJ}$ \\
\hline 23 & Zheng et al. (2003) & IR1552/Azucena & RILs F10 & $\mathrm{I} / \mathrm{TJ}$ \\
\hline 24 & Zheng et al. (2000) & IR64/Azucena & $\mathrm{DH}$ & $\mathrm{I} / \mathrm{TJ}$ \\
\hline
\end{tabular}

populations were mostly derived from indica/japonica or japonica/indica crosses (eight populations representing 560 QTLs) for polymorphism reasons. The set also included two indica $\times$ indica populations (91 QTLs), two temperate japonica/tropical japonica populations (21 QTLs), and one temperate japonica/aus population (three QTLs). Some traits such as MRL or RTHK were measured in a large number of studies (13 and 12, respectively), while other traits such as lateral root length or deep root number were measured in just one study. For each trait, Table 3 presents an overview of the number of QTLs in segments of $5.0 \mathrm{Mb}$ covering the whole genome. QTLs were found in almost all segments with an average of 8.4 QTLs per segment, but a concentration of above 20 QTLs was observed in six segments, namely, the $30-$ to $40-\mathrm{Mb}$ segment of chromosome 1 , the $25-$ to $35-\mathrm{Mb}$ interval of chromosome 2; the 0 to $5-\mathrm{Mb}$ interval of chromosome 3 , the $30-$ to $35-\mathrm{Mb}$ interval of chromosome 4 , the $15-$ to $20-\mathrm{Mb}$ interval of chromosome 9 , and the $15-$ to $20-\mathrm{Mb}$ interval of chromosome 11 . These segments, which represented $10 \%$ of the genome in length, carried $30 \%$ of the total number of QTLs.

Supplementary Figure 1 presents the chromosomal location of the QTLs. Traits for which less than 20 QTLs were detected, of least interest for the meta-QTL analysis, are not included in the figure for reasons of space. The last letter of the QTL name indicates under which conditions the QTL was detected, with " $w$ " standing for well-watered conditions (368 among the 675 QTLs), "s" standing for water stress conditions (147 QTLs), and "c" for compaction stress using the wax petrolatum layer system (160 QTLs). For the traits for which the number of QTLs detected in both control and stressed conditions permitted binary comparisons [MRL, RTHK for well-watered and water stress conditions; RN versus PRN, RTHK versus penetrated root thickness (PRTHK) for standard and compacted conditions], the QTLs observed under stressed conditions were generally co-localized with QTLs detected under favorable conditions notably in the six QTL hot spot areas mentioned above. Several isolated QTLs specific to one condition or another were also found (Supplementary Figure 2). Some clusters grouping only QTLs detected under well-watered conditions were also observed.

Meta-QTL analysis

Meta-QTL analysis was possible for ten root traits $(\mathrm{RN}$, PRN, MRL RTHK, PRTHK, RDW, DRW, DRR, DR/T, and 
Table 2 Root Parameters for Which QTLs Were Detected

\begin{tabular}{|c|c|c|c|c|}
\hline Trait name & Symbol & $\mathrm{Nb}$ populations & Nb QTLs & Reference of the studies in Table1 \\
\hline Root number & $\mathrm{RN}$ & 6 & 41 & $1,4,8,12,14,23,24$ \\
\hline Deep root number & DRN & 1 & 11 & 13 \\
\hline Lateral root number & LRN & 1 & 3 & 23 \\
\hline Penetrated root number & PRN & 4 & 25 & $1,12,14,24$ \\
\hline Seminal root length & SRL & 1 & 7 & 23 \\
\hline Maximum root length & MRL & 10 & 103 & $3,4,5,6,7,9,11,13,15,18,19,20,22$ \\
\hline Lateral root length & LRL & 1 & 5 & 23 \\
\hline Penetrated root length & PRL & 2 & 7 & $1,10,21$ \\
\hline Root thickness & RTHK & 7 & 123 & $2,3,4,6,7,8,9,10,11,13,19,21$ \\
\hline Penetrated root thickness & PRTHK & 3 & 37 & $1,10,21,24$ \\
\hline Root volume & RVOL & 2 & 18 & 4,20 \\
\hline Root dry weight & RDW & 6 & 38 & $3,4,5,8,10,18,19,21$ \\
\hline Root dry weight in the $00-30 \mathrm{~cm}$ layer & RW0030 & 1 & 5 & 2 \\
\hline Root dry weight in the $30-60 \mathrm{~cm}$ layer & RW3060 & 1 & 3 & 2 \\
\hline Root dry weight in the $60-90 \mathrm{~cm}$ layer & RW6090 & 1 & 2 & 2 \\
\hline Root fresh weight & RFW & 1 & 4 & 8 \\
\hline Penetrated root dry weight & PRDW & 1 & 7 & 10,21 \\
\hline Deep root weight & DRW & 5 & 42 & $3,6,7,9,13,19$ \\
\hline Deep root ratio & DRR & 3 & 31 & $6,7,20$ \\
\hline Root to total biomass ratio & $\mathrm{R} / \mathrm{BIOM}$ & 1 & 16 & 9 \\
\hline Root to shoot ratio & $\mathrm{R} / \mathrm{S}$ & 4 & 30 & $2,8,13,18$ \\
\hline Deep root to shoot ratio & $\mathrm{DR} / \mathrm{S}$ & 2 & 7 & 3,19 \\
\hline Root dry weight per tiller & $\mathrm{R} / \mathrm{T}$ & 1 & 14 & 2 \\
\hline Deep root weight per tiller & $\mathrm{DR} / \mathrm{T}$ & 5 & 22 & $2,3,6,7,19$ \\
\hline Root branching index & RBI & 1 & 3 & 5 \\
\hline Root growth rate in depth & RGRD & 1 & 14 & 20 \\
\hline Root growth rate in volume & RGRV & 1 & 19 & 20 \\
\hline Root penetration index & RPI & 5 & 35 & $1,10,12,14,21,24$ \\
\hline Root pulling force & RPF & 1 & 12 & 10,21 \\
\hline Total number of root QTLs & & & 675 & \\
\hline
\end{tabular}

RPI) with sufficient QTL density (more than five) on at least one of the 12 chromosomes. Therefore, only 306 QTLs out of the 675 originally identified, representing a total of 32 root trait $\times$ chromosome combinations, were submitted to the meta-analysis. A total of 119 meta-QTLs were detected. The complete list of the 119 meta-QTLs, with their physical position as well as the list of the 306 QTLs, is given in Supplementary Table 2. Graphical results of the meta-QTL analysis are presented in Supplementary Figure 3 . In each trait $\times$ chromosome figure, the lengths of the colored segments in each QTL bar are proportional to their probability of belonging to the meta-QTL of the same color. The probability for a QTL to belong to a cluster is indicated in Supplementary Table 2. Most of the QTLs (93.0\%) were attributed to just one cluster with a probability of more than $90 \%$. The QTLs with a probability of attribution to several clusters were those with the longest confidence intervals, which were generally found in the doubled haploid or backcross mapping populations.

Extracts of Supplementary Table 2 and Supplementary Figure 3 focusing on MRL and chromosomes 1 and 9 are given as examples in Table 4 and Figs. 1 and 2.

The 119 meta-QTLs detected corresponded to a reduction of $61 \%$ in the number of QTLs. The reduction was not evenly distributed. On some chromosomes such as chromosome 3 for RTHK, the QTLs were distributed in such a way that little was gained by meta-analysis (ten QTLs resolved into six meta-QTLs). Some QTLs remained isolated. On some others, QTLs were concentrated on a short segment and the effect of the meta-analysis was striking. Good examples are the $30-$ to $40-\mathrm{Mb}$ segment of 
Table 3 Number of QTLs and Meta-QTL for Diverse Root Traits in 5-Mb Windows

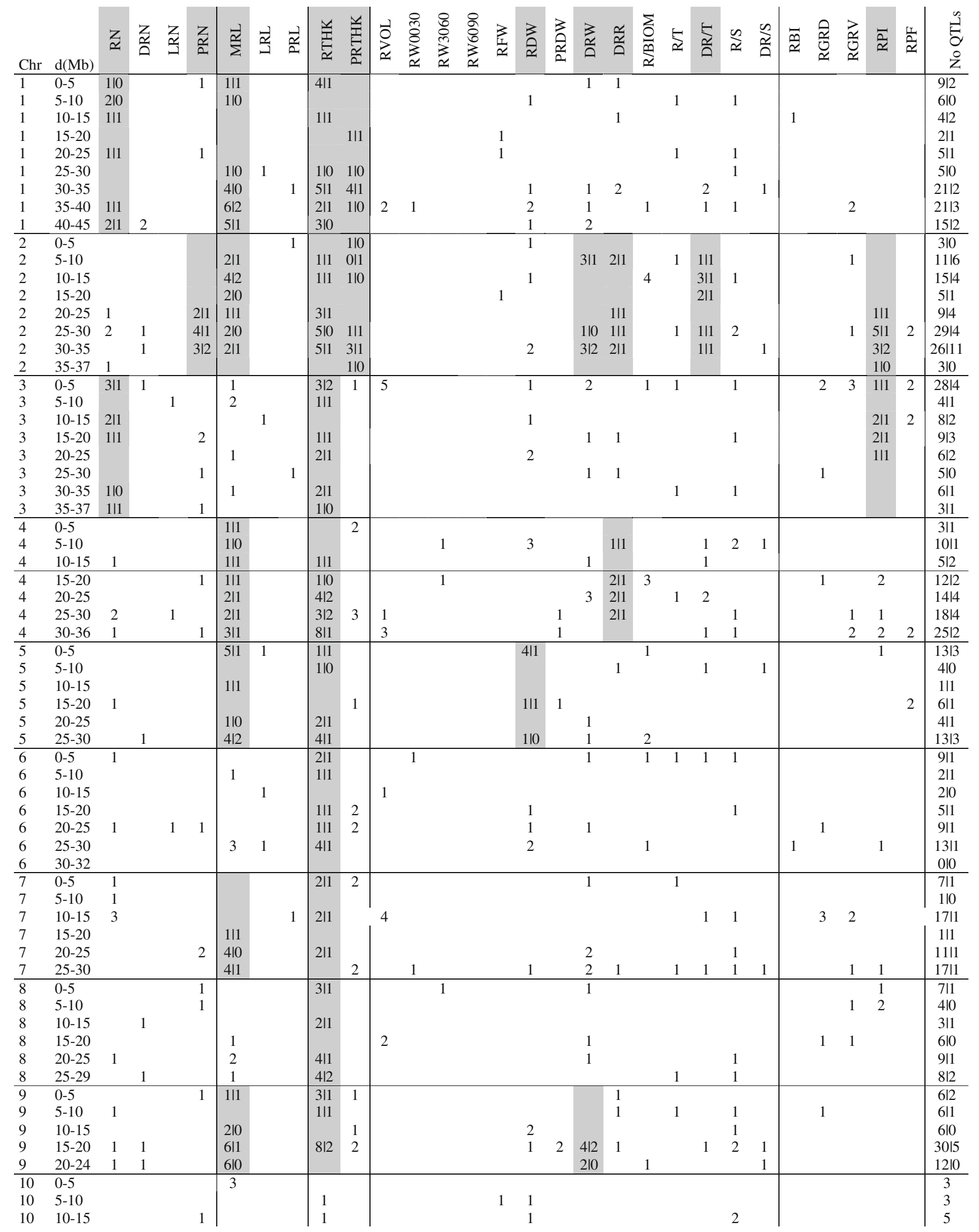


Table 3 (continued)

\begin{tabular}{|c|c|c|c|c|c|c|c|c|c|c|c|c|c|c|c|c|c|c|c|c|c|c|c|c|c|c|c|c|c|c|}
\hline Chr & $\mathrm{d}(\mathrm{Mb})$ & Z & 瓷 & Z & $\underset{\mathfrak{n}}{\underline{2}}$ & $\overrightarrow{\widetilde{\alpha}}$ & $\vec{a}$ & $\vec{\alpha}$ & 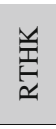 & $\begin{array}{l}\text { 兰 } \\
\text { 点 } \\
\text { 点 }\end{array}$ & $\stackrel{D}{a}_{a}$ & 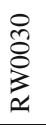 & 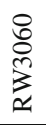 & $\begin{array}{l}8 \\
8 \\
0 \\
3 \\
3\end{array}$ & $\frac{3}{x}$ & ลิ & 芫 & 党 & $\frac{a}{\frac{a}{a}}$ & $\frac{\sum}{0}$ & 5 & $\sum_{0}^{2}$ & $\frac{\tilde{2}}{\approx}$ & $\frac{n}{2}$ & $\vec{a}$ & 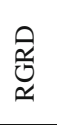 & 总 & $\overline{\widetilde{a}}$ & $\frac{1}{2}$ & $\begin{array}{l}3 \\
\hat{1} \\
0 \\
z\end{array}$ \\
\hline $\begin{array}{l}10 \\
10\end{array}$ & $\begin{array}{l}15-20 \\
20-24\end{array}$ & & & & & 1 & & & & 1 & & & & 1 & & 2 & & & 1 & & & & & & & & & 1 & & $\begin{array}{l}5 \\
2\end{array}$ \\
\hline 11 & $0-5$ & & & & & 311 & & & 111 & & & & & & & 2 & & 1 & $2 \mid 1$ & & & & & & & 1 & & 1 & & 1113 \\
\hline 11 & $5-10$ & 1 & & & & & & & & & & & & & & & & & & & & 1 & & & & & & & & $\begin{array}{l}210 \\
610\end{array}$ \\
\hline 11 & $10-15$ & & & & & & & & & & & & & & & 1 & & & & & & & & & 1 & & 4 & & & 610 \\
\hline 11 & $15-20$ & 1 & & & & 111 & & 1 & 311 & & & 1 & & 1 & & 1 & & 3 & 311 & & & & 1 & & & 2 & & 1 & 2 & 2113 \\
\hline 11 & $20-25$ & 2 & & & 1 & 311 & & & 311 & & & & & & & & & & 110 & 1 & & & & & & & & 1 & & 1212 \\
\hline 11 & $25-31$ & & & & & & & 2 & 110 & & & & & & & & & & & & & & 1 & & & & & & & 410 \\
\hline 12 & $0-5$ & & 1 & & & & & & 211 & & & & & & & & & & & & & & & & & & & & & $3 \mid 1$ \\
\hline 12 & $5-10$ & 1 & & & 1 & & & & $2 \mid 1$ & & & & & & & & & & & & & & 1 & & & & & & & $5 \mid 1$ \\
\hline 12 & $10-15$ & & & & & & & & & & & & & & & & & & & & & & & & & & & & & $0 \mid 0$ \\
\hline 12 & $15-20$ & & & & & & & & & 2 & & & & & & & 2 & & & & 1 & & & & & 1 & & 2 & & 810 \\
\hline 12 & $20-25$ & & & & & & & & $2 \mid 1$ & 1 & & & & & & & & & & & & & & & & & & & & $3 \mid 1$ \\
\hline 12 & $25-28$ & & & & & 2 & & & 110 & & & 1 & & & & & & & & & 1 & & & & & & & & & 510 \\
\hline Tot & QTLs & 41 & 11 & 3 & 25 & 103 & 5 & 7 & 123 & 37 & 18 & 5 & ? & 2 & 4 & 38 & 7 & 42 & 3 & 16 & 14 & 22 & 30 & 7 & 3 & 14 & 19 & 33 & 12 & 675 \\
\hline No $C$ & TLs $^{1}$ & 16 & 0 & 0 & 9 & 84 & 0 & 0 & 121 & 14 & 0 & 0 & 0 & 0 & 0 & 6 & 0 & 13 & 19 & 0 & 0 & 8 & 0 & 0 & 0 & 0 & 0 & 16 & 0 & 306 \\
\hline No $n$ & $\mathrm{CQTs}^{2}$ & 8 & 0 & 0 & 4 & 26 & 0 & 0 & 46 & 5 & 0 & 0 & U & 0 & 0 & 2 & 0 & 5 & 10 & . & 0 & 5 & 0 & 0 & 0 & 0 & 0 & 8 & 0 & 119 \\
\hline
\end{tabular}

In gray, trait $\times$ chomosome combinations submitted to meta-analysis. In these columns, the number of QTLs and the number of meta-QTLs are indicated on the left and right sides of the "I", respectively

Trait symbols according to Table 2. Chromosome lengths based on Nipponbare pseudo-molecule release 5.0.

1: Number of QTLs involved in the meta-analysis; 2: Number of meta-QTLs

chromosome 1 carrying 15 QTLs for MRL resolved into three QTLs (Fig. 1) or the 10- to 23-Mb segment of chromosome 9 carrying 14 QTLs for MRL resolved into just one (Fig. 2).

As for resolution power, the increase in precision of the QTL location was also strong. The average confidence interval of the QTLs involved in the meta-analysis was $4.14 \mathrm{Mb}$ and went down to 1.98 for the meta-QTLs. The meta-QTL confidence interval was generally smaller than the smallest confidence interval of the QTLs of the cluster. This was all the more true when the cluster encompassed a large number of QTLs.

Twenty-five meta-QTLs had a confidence interval below $250 \mathrm{~kb}$, small enough to envision browsing the underlying genes. Six of the meta-QTLs on chromosome 2 corresponded almost exactly to the same physical interval, which reduced the number of different meta-QTLs to 20. The genes located in these segments were retrieved from the Nipponbare sequence. Those with an indication of biological function are listed in Supplementary Table 3. The splice variants of a given gene were not individualized. The number of genes with a known function underlying the meta-QTLs varied from 0 to 32. Among those that could be of particular interest in the context of root development (in bold in Supplementary Table 3), we observed genes involved in auxin signaling and transport, transcription factors, and sugar metabolism genes.

Comparison between the positions of the meta-QTLs and those of three cloned genes controlling flowering time showed that for $H d 3 a$ and $H d l$ on chromosome 6, the confidence interval of the meta-QTLs mqDUR6-2 and
mqDUR6-3 included the gene positions (Supplementary Figure 3). This remained true when the QTL with the longest confidence interval was removed from the analysis, which resulted in a sharp decrease in the confidence interval of the meta-QTLs. On chromosome 3, the two meta-QTLs detected in the region clearly did not overlap with Hd6, but nor did most of the QTLs in this zone. For the analysis of plant height QTLs on chromosome 1, one of the meta-QTLs, mqHGT1-4, was close to $S d 1$, but still $340 \mathrm{~kb}$ away, while some of the QTL confidence intervals included the gene position (Supplementary Figure 3). The QTLs for MRL on the distal part of chromosome 1, which have a major effect, have often been said to correspond to $S d 1$, assuming a pleiotropic effect of the semi-dwarfism gene. $S d 1$ was indeed within the confidence interval of the meta-QTL mqMRL_1-4 for MRL.

\section{Discussion}

We constructed a database of drought QTLs in rice that assembles a large body of information on each QTL (http:// tropgenedb.cirad.fr/en/rice.html). These QTLs can be visualized on an integrative map using a map viewer. Complex queries on various fields including the experimental conditions under which the QTLs were detected are possible directly on the web site. Another convenient option is to download a subsample of QTLs in an Excel format file and filter it on any of the available fields. This procedure is particularly useful for immediate assessment of the presence of a QTL in a given physical zone by filtering 
Table 4 QTLs Reported and Meta-QTLs Detected for Maximum Root Length on Chromosomes 1 and 9

\begin{tabular}{|c|c|c|c|c|c|c|c|c|}
\hline Chr. & QTL name & Type & Position $^{\mathrm{a}}$ & CI & $\mathrm{M} 1^{\mathrm{b}}$ & M2 & M3 & M4 \\
\hline 1 & MacMillan_2006_1538s & Q & 1.27 & $0.112-2.424$ & 1 & 0 & 0 & 0 \\
\hline 1 & mqMRL_1-1 & M & 1.76 & $0.655-2.865$ & 1 & 0 & 0 & 0 \\
\hline 1 & Horii_2006_1824w & Q & 7.09 & $3.279-10.904$ & 1 & 0 & 0 & 0 \\
\hline 1 & Yadav_1997_166w & Q & 29.32 & $22.404-36.242$ & 0 & 0.90 & 0.09 & 0.01 \\
\hline 1 & Price_1999_1621s & Q & 30.58 & $28.677-32.473$ & 0 & 1 & 0 & 0 \\
\hline 1 & mqMRL_1-2 & M & 32.50 & $31.625-33.375$ & 0 & 1 & 0 & 0 \\
\hline 1 & Price_1999_1614w & Q & 32.57 & $31.280-33.856$ & 0 & 1 & 0 & 0 \\
\hline 1 & Kamoshita_2002b_1012w & Q & 33.59 & $31.597-35.590$ & 0 & 1 & 0 & 0 \\
\hline 1 & Courtois_2003_107w & Q & 33.95 & $31.647-36.258$ & 0 & 0.98 & 0.02 & 0 \\
\hline 1 & MacMillan_2006_1533w & Q & 35.71 & $34.160-37.263$ & 0 & 0.01 & 0.99 & 0 \\
\hline 1 & Zheng_2003_510s & Q & 37.17 & $35.410-38.921$ & 0 & 0 & 1 & 0 \\
\hline 1 & Zheng_2003_509w & Q & 37.17 & $35.333-38.999$ & 0 & 0 & 1 & 0 \\
\hline 1 & mqMRL_1-3 & M & 37.70 & $36.255-39.145$ & 0 & 0 & 1 & 0 \\
\hline 1 & Price_2002c_1758s & Q & 38.40 & $37.276-39.522$ & 0 & 0 & 1 & 0 \\
\hline 1 & MacMillan_2006_1539s & Q & 38.40 & $36.170-40.628$ & 0 & 0 & 0.81 & 0.19 \\
\hline 1 & Zhang_2001b_1086w & Q & 38.86 & $37.533-40.185$ & 0 & 0 & 0.79 & 0.21 \\
\hline 1 & MacMillan_2006_1534w & Q & 40.28 & $39.718-40.842$ & 0 & 0 & 0 & 1 \\
\hline 1 & MacMillan_2006_1543w & Q & 40.28 & $39.406-41.154$ & 0 & 0 & 0 & 1 \\
\hline 1 & Price_2002c_1768s & Q & 40.28 & $39.098-41.462$ & 0 & 0 & 0 & 1 \\
\hline 1 & mqMRL_1-4 & M & 40.43 & $40.245-40.615$ & 0 & 0 & 0 & 1 \\
\hline 1 & Redona\&Mackill_1996_1212w & Q & 41.07 & $40.082-42.060$ & 0 & 0 & 0 & 1 \\
\hline 1 & Redona\&Mackill_1996_1211w & Q & 41.07 & $39.862-42.280$ & 0 & 0 & 0 & 1 \\
\hline 9 & Yue_2006_1297w & Q & 4.48 & $3.041-5.923$ & 1 & 0 & & \\
\hline 9 & mqMRL_9-1 & M & 5.40 & $4.075-6.725$ & 1 & 0 & & \\
\hline 9 & Kamoshita_2002a_970w & Q & 10.06 & $6.808-13.315$ & 1 & 0 & & \\
\hline 9 & Kamoshita_2002a_975w & Q & 14.80 & $8.682-20.928$ & 0 & 1 & & \\
\hline 9 & Horii_2006_1825w & Q & 17.01 & $11.467-22.558$ & 0 & 1 & & \\
\hline 9 & Price_1999_1625s & Q & 18.65 & $17.543-19.760$ & 0 & 1 & & \\
\hline 9 & MacMillan_2006_1541s & Q & 19.03 & $17.870-20.186$ & 0 & 1 & & \\
\hline 9 & Price_2002c_1764s & Q & 19.03 & $17.050-21.006$ & 0 & 1 & & \\
\hline 9 & Price_2002c_1775s & Q & 19.52 & $18.861-20.188$ & 0 & 1 & & \\
\hline 9 & mqMRL_9-2 & M & 19.61 & $19.590-19.630$ & 0 & 1 & & \\
\hline 9 & Yadav_1997_171w & Q & 19.95 & $12.955-26.951$ & 0 & 1 & & \\
\hline 9 & MacMillan_2006_1537w & Q & 20.12 & $18.125-22.124$ & 0 & 1 & & \\
\hline 9 & Zheng_2003_514s & Q & 20.21 & $18.669-21.760$ & 0 & 1 & & \\
\hline 9 & Zheng_2003_515w & Q & 20.21 & $18.724-21.705$ & 0 & 1 & & \\
\hline 9 & Yue_2006_1300w & Q & 20.49 & $18.078-22.899$ & 0 & 1 & & \\
\hline 9 & Yue_2006_1305s & Q & 20.49 & $19.136-21.842$ & 0 & 1 & & \\
\hline 9 & Courtois_2003_108w & Q & 21.34 & 19.399-23.279 & 0 & 1 & & \\
\hline
\end{tabular}

$M$ meta-QTL, $Q$ QTL, $C I$ confidence interval on the position

${ }^{\text {a }}$ Position in megabases

${ }^{\mathrm{b}} \mathrm{M} 1$ to M4: probability that the QTL belongs to a given cluster

the locus position field for a range of physical positions. Conversely, existing bioinformatics tools such as those available in OrygenesDB (http://orygenesdb.cirad.fr/) can be used to list the genes underlying any QTL confidence interval (Droc et al. 2006).
The compilation presented here focuses on root traits, but the same work is possible for any other drought resistance trait such as leaf rolling score, leaf drying score, or osmotic adjustment for which most QTLs from the 1995-2007 period have been compiled in the database. The 

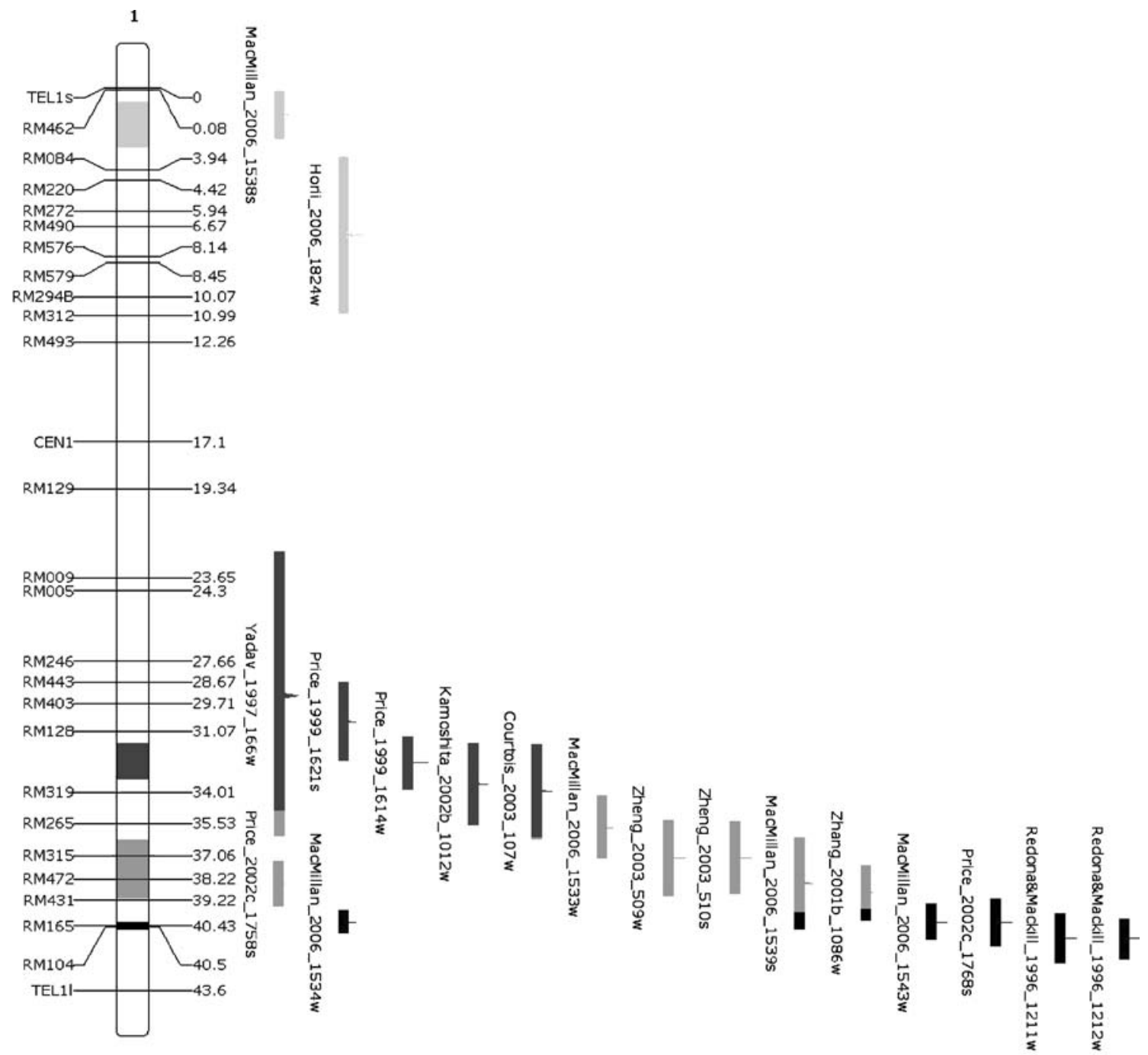

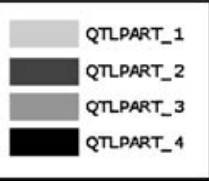

Fig. 1 QTL and meta-QTLs for maximum root length on chromosome 1. The meta-QTLs on the body of the chromosome are represented by bars of different intensities of gray. The lengths of the gray segments in each QTL bar are proportional to their

database module will be yearly updated with integration of the newly published QTLs.

The projection of all QTLs on a virtual map allows the issue of whether the QTLs detected under stress conditions are or are not a subset of those detected under favorable conditions to be addressed. In the case of root traits, QTLs detected under stress conditions co-localized with QTLs detected under favorable conditions in the majority of the cases. The possibility that different QTLs (and presumably the underlying genes) expressed in the different situations probability of belonging to the meta-QTL of the same intensity of gray. Distances on the map are in megabases. A color version of the figure can be found in Supplementary Figure 3.

can be clustered in the same region cannot be excluded, but more likely, the same QTL (and underlying gene) works differently depending on the growth conditions, as demonstrated by MacMillan et al. (2006). For the few clusters grouping only QTLs detected in one condition, the more frequent presence of clusters grouping only QTLs expressed under favorable conditions can be explained by the larger number of QTLs of this type, associated with the higher heritability of traits measured under favorable conditions. The detection of QTLs in some populations and not in others 


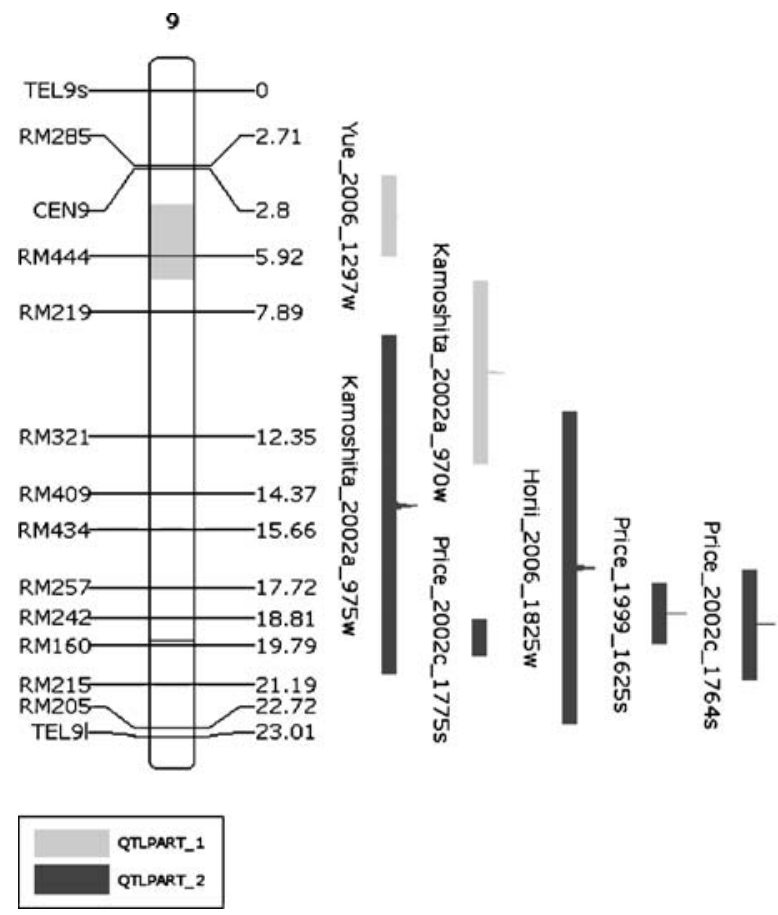

Fig. 2 QTL and meta-QTLs for maximum root length on chromosome 9. The meta-QTLs are represented by bars of different intensities of gray on the body of the chromosome. Distances on the map are in

may be due to the segregation of the underlying genes in various mapping populations and fixation of the same genes in other populations.

We ran the meta-analysis separately for each trait because the method normally requires the experiments to be independent. Nevertheless, it is possible to pool different correlated traits measured in the same individuals in a single meta-analysis since Goffinet and Gerber (2000) showed through simulation that the procedure was robust even in such cases. Assuming that several of the traits studied are pleiotropically related, analysis of pooled traits may be more powerful than analysis of an individual trait. It may also increase the number of chromosomes with more than six QTLs and therefore ensure more complete coverage of the genome than the 32 trait $\times$ chromosome combinations we studied. The pooled trait approach is presently being applied to the root QTLs of the Azucena $\times$ Bala population (Khowaja et al. 2009). Time will tell which approach was best when the genes are cloned. Meanwhile, one would expect that the analysis of pooled traits works best for pleiotropy situations but less well for tight linkage.

The global QTL dataset we submitted to meta-analysis was not as precise as each of the individual QTL mapping studies we compiled. This was unavoidable because of (1) the intrinsic need of the meta-QTL analysis tool for homogeneous data, (2) the heterogeneity of the source data, and (3) some of our methodological choices. Information on the precise positions or confidence megabases. A color version of the figure can be found in Supplementary Figure 3.

intervals of the putative QTLs, for example, was often absent from the papers, with just indication of the flanking markers. Our decision to use a proxy for non-sequenced markers led to reduced precision concerning the position of QTLs linked to these markers. Also, the ordering of the markers on some genetic maps can be locally different from their order on physical maps. If the problem is inconsequential with single marker analysis, the impact of map errors on QTL position is likely to have increased with the shift to simple or composite interval mapping analyses. The information set that we were finally able to use was therefore reduced to the minimum common denominator when all papers were taken into account. Another option would be to exclude the least informative papers from the analysis.

Offsetting its need for a homogeneous QTL dataset, meta-QTL analysis allows an advance over browsing the database by reducing the number of observed QTLs to a more limited number of meta-QTLs with a narrower confidence interval. The best example is probably the meta-QTL for MRL on chromosome 9 whose confidence interval was reduced to a length of $20 \mathrm{~kb}$, thereby reducing resolution to a few genes (two genes in this interval). At first glance, however, neither of the two genes seemed to be a good candidate for root system development, but six genes away (a distance of $40 \mathrm{~kb}$ ), there was an auxin efflux carrier that was a better candidate based on its function. It thus appears that the reduction in the confidence interval 
may be very important, but these results should be considered with caution in such cases. For other QTL clusters, the gain was more modest since the average confidence interval of the meta-QTLs was close to $2 \mathrm{Mb}$, which, in rice, represents an average of 200 genes. MetaQTL analysis works better with a large number of QTLs and gives less reliable results when the number of QTLs per chromosome is below six. But this is also the situation in which meta-QTL analysis is the least interesting because the QTLs are less widely distributed among the mapping populations, or have smaller effects.

We evaluated the efficiency of meta-QTL analysis in improving the prediction of the meta-QTL positions by applying the method to QTLs surrounding four cloned genes. On chromosome 6, which carries the $H d 1$ and $H d 3$ flowering time genes, precision was considerably improved since the confidence intervals of the two meta-QTLs colocalizing with the genes were smaller than that of each individual QTL mapped in the individual studies we analyzed. But a third meta-QTL was also detected. On chromosome 3, however, the meta-QTLs did not cover the Hd6 gene, but nor did most of the QTLs, so it is likely that another gene located closer to the top of chromosome 3 is responsible for the meta-QTL for flowering time detected there. On chromosome 1, the $S d 1$ gene controlling plant height was very close to the meta-QTL with the narrowest confidence interval of the three meta-QTLs detected in this area. The number of cloned QTLs used for this validation was low, and this result may be a coincidence. Moreover, both flowering time and plant height have higher broad sense heritability than root traits, and QTLs for root traits will have been located with less precision than those used in the evaluation of the efficiency of the method. In addition, meta-QTL analysis frequently identified several meta-QTLs under a QTL cluster. It is possible that each meta-QTL corresponds to a different gene, but the situation may also reflect a bias in the method. Nevertheless, all things considered, these results make us confident that the method described here is valuable, probably all the more so when the QTL dataset is of high quality.

There are several possible ways to go further in the identification of candidate genes, the highest efficiency being expected from a combination of options producing a range of evidence. For the meta-QTLs with the longest confidence intervals, the identified regions can be explored by association mapping of appropriate genotype panels and a denser set of markers for the region concerned, enabling the QTL position to be narrowed down. The method can be used for segments longer than $500 \mathrm{~kb}$, which is the upper limit of linkage disequilibrium span in rice (Mather et al. 2007), and shorter than $4 \mathrm{Mb}$, which is the average QTL confidence interval in our dataset and the point at which association mapping will not provide added value compared to bi- parental population mapping. For meta-QTL with a confidence interval below $500 \mathrm{~kb}$, the underlying candidate genes can easily be listed. Under our meta-QTLs, we found, for example, genes involved in auxin signaling and transport or in cell division, transcription factors expressed under abiotic stresses, and sugar metabolism genes that could act on root growth or serve as osmoprotectants. However, all genes within the confidence interval could be candidates for further work, and those with no known function could not be eliminated either. Links between the gene function and the QTL can be reinforced by the involvement of the gene in a relevant pathway such as those described by Malamy (2005), Nibau et al. (2008), or Rebouillat et al. (2008) for root development or by the expression of the gene in root tissues, a method explored by Norton et al. (2008). Mutants with tDNA insertion in the candidate genes can also be searched in OrygenesDB, their expression in root tissues checked by using Oryza-Tag Line GUS and GFP data (Larmande et al. 2008) and the mutants analyzed to check for the mutation effect on root morphology or development.

However, it is important to note that even without knowing the genes involved, the reduction both in the number of QTLs and size of their confidence interval should greatly facilitate the manipulation of the QTL carrying segments through marker-assisted selection. The scheme to build improved genotypes will be all the more complex if the number of segments to manipulate is large (Hospital and Charcosset 1997). For a limited number of candidate genes, one possible approach is to use the genotype building strategy proposed by Servin et al. (2004), focusing for example on the six zones already identified as potentially interesting in this paper. But the chances are that the interesting alleles with large effects will already be present in material with good drought resistance and that breeders will have to manipulate a larger number of QTLs with smaller effects. Additional progress leading to the accumulation of these QTLs can then be envisioned through marker-aided recurrent selection (Hospital et al. 2000; Bernardo and Charcosset 2006).

\section{Methods}

\section{Statistical methods}

The first element needed to run a meta-analysis is a single integrative map where all markers mentioned in the different studies are positioned. From the data available in the papers, it was not possible to establish a consensus genetic map since the genetic distance between markers was not indicated for all the mapping populations. Consequently, we chose to use the marker order of the physical map and the physical distance between markers as explained above. 
The second element required is the confidence intervals of the QTL. In some papers, the confidence interval was computed using a support interval that was either LODmax1 or LODmax-2, but in most papers, the confidence interval was not given. To homogenize the data and estimate missing confidence intervals, a simpler solution was to use the empirical formula proposed by Darvasi and Soller (1997) for $F_{2}$ and BC and extended by Guo et al. (2006) to other population types. For a $\mathrm{BC}$, an $\mathrm{F}_{2}$ or a $\mathrm{DH}$ population, the QTL confidence interval at the $95 \%$ level is $530 / \mathrm{Nr}^{2}$, where $N$ is the population size and $r^{2}$ is the proportion of the total variance explained by the QTL. For a RIL population, the formula becomes $163 / \mathrm{Nr}^{2}$. These formulas generated confidence interval limits in centimorgan (cM). We converted these values into physical positions using a ratio of $1 \mathrm{cM}$ per $244 \mathrm{~kb}$ (Chen et al. 2002) assuming that the ratio of genetic to physical distance was invariant throughout the genome. It is known that the correspondence between physical and genetic maps is not homogeneous along the chromosomes. But, in rice, unlike in maize, barley, and wheat, the reduced frequency of recombination mostly affects the areas surrounding the centromeres, and recombinationally inactive regions are limited to a few megabases (Chen et al. 2002). The bias the assumption is likely to introduce in the analyses will be concentrated in the centromeric regions, but in our dataset, the QTLs located in the $5-\mathrm{Mb}$ window around the centromeres (2.5 $\mathrm{Mb}$ each side) represented only $9.1 \%$ of the total number of QTLs.

The meta-QTL analysis was run using the software package MetaQTL (Veyrieras et al. 2007). The method relies on a clustering algorithm based on a Gaussian mixture model and enables determination of the likely number of clusters considered as the "true" QTLs underlying the QTLs observed in a given region. The optimal number of clusters is chosen by means of an informationbased criterion, and the position and confidence interval of the meta-QTLs can then be estimated. Meta-QTL proposes five different criteria for model choice, but here, we present only the results based on the Akaike information content criterion, which performed well in simulations (Veyrieras et al. 2007).

Meta-analysis was not attempted for chromosomes carrying less than six QTLs, as in this case, the model performed poorly because of over-parameterization.

To facilitate the visualization of the QTLs and metaQTLs and the shift back to markers, the QTLs and metaQTLs were projected on a virtual physical map composed of microsatellite markers corresponding to those used to build the IR64xAzucena SSD genetic map (Ahmadi et al., unpublished data). The distances between markers mentioned on the map are physical distances expressed in megabases.
The genes underlying the meta-QTLs were extracted with the tool "Get sequence" of OrygenesDB (http:// orygenesdb.cirad.fr) applied to the Nipponbare sequence (Droc et al. 2006).

\section{Evaluation of efficiency of meta-QTL analysis}

A comparison of the position of consensus QTLs obtained by meta-analysis with that of cloned genes for the same trait was performed to evaluate the efficiency of the method. No QTL for root trait has yet been cloned, but the approach was possible for flowering time and plant height. A total of 80 QTLs for flowering time detected under well-watered conditions were collected from ten papers ( $\mathrm{Li}$ et al. 1995; Yano et al. 1997; Lafitte and Courtois 1999; Lin et al. 2000; Maheswaran et al. 2000; Yamamoto et al. 2000; Yu et al. 2002; Hittalmani et al. 2003; Lanceras et al. 2004; Dong et al. 2004). A total of 59 QTLs for plant height were collected from ten papers ( $\mathrm{Li}$ et al. 1995; Lafitte and Courtois 1999; Hemamalini et al. 2000; Yu et al. 2002; Courtois et al. 2003; Hittalmani et al. 2003; Lanceras et al. 2004; Xu et al. 2004; MacMillan et al. 2006; Gomez et al. 2006). All the QTLs are compiled in TropgeneDB. These QTL were submitted to a meta-QTL analysis in the same way as the root QTLs. The meta-QTL positions for flowering time were then compared to the physical position of three cloned flowering time QTLs: $H d l$ cloned by Yano et al. (2000) and Hd3a cloned by Kojima et al. (2002), both on chromosome 6 , and $H d 6$ cloned by Takahashi et al. (2001) on chromosome 3. These QTLs corresponded to genes Os06g16370 located between base pairs 9,335,361 and 9,337,634 on chromosome 6, Os06g06320 located between bp 2,939,005 and 2,941,453 on chromosome 6 and Os03g10940 located between base pairs 5,595,162 and $5,599,842$ on chromosome 3 , respectively. The same comparison was undertaken for the meta-QTLs for plant height on chromosome 1 whose positions were compared to that of $S d 1$ (Os01g66100) cloned by Monna et al. (2000) located between base pairs 38,709,231 and 38,712,353.

Acknowledgments The Generation Challenge Program partly funded this work through the project "Comparative QTL mapping for drought tolerance" (no. 2005-17). We acknowledge also the support of the "European Commission funded project no. 015468 CEDROME" which funded F. Khowaja's stay in Cirad-Montpellier. We thank J-B Veyrieras for his answers on the best way to use MetaQTL. We are grateful to Vanina Lenieff and Raphaël de Valavielle for helping in assembling the dataset. We thank Pankaj Jaiswal, from Gramene, for his comments on the trait definitions and the associated ontology.

Open Access This article is distributed under the terms of the Creative Commons Attribution Noncommercial License which permits any noncommercial use, distribution, and reproduction in any medium, provided the original author(s) and source are credited. 


\section{References}

Ali ML, Pathan MS, Zhang J, Bai G, Sarkarung S, Nguyen HT. Mapping QTLs for root traits in a recombinant inbred population from two indica ecotypes in rice. Theor Appl Genet. 2000;101:756-66.

Arcade A, Labourdette A, Falque M, Mangin F, Chardon F, Charcosset A, et al. BioMercator: integrating genetic maps and QTLs toward discovery of candidate genes. Bioinformatics. 2004;20:2324-6.

Bernardo R, Charcosset A. Usefulness of gene information in markerassisted recurrent selection: a simulation appraisal. Crop Sci. 2006;46:614-21.

Champoux C, Wang G, Sarkarung S, Mackill OJ, O'Toole JC, Huang $\mathrm{N}$, et al. Locating genes associated with root morphology and drought avoidance in rice via linkage to molecular markers. Theor Appl Genet. 1995;90:969-81.

Chardon F, Virlon B, Moreau L, Falque M, Joets J, Decousset L, et al. Genetic architecture of flowering time in maize as inferred from quantitative trait loci meta-analysis and synteny conservation with the rice genome. Genetics. 2004;168:2169-85.

Chen M, Presting G, Barabzuk WB, Goicoechea JL, Blackmo B, Fang G, et al. An integrated physical and genetic map of the rice genome. Plant Cell. 2002;14:537-45.

Coque M, Martin A, Veyrieras JB, Hirel B, Gallais A. Genetic variation for $\mathrm{N}$-remobilization and post-silking $\mathrm{N}$-uptake in a set of maize recombinant inbred lines. 3. QTL detection and coincidences. Theor Appl Genet. 2008;117:729-47.

Courtois B, Chaitep W, Moolsri S, Sinha PK, Trebuil G, Yadav R. Drought resistance and germplasm improvement. On-going research in the Upland Rice Research Consortium. In: Piggin C, Courtois B, Schmit V, editors. Upland rice research in partnership, IRRI, Los Baños, Philippines. IRRI Discussion Paper Series 16;1996. p. $154-75$

Courtois B, Shen L, Petalcorin W, Carandang S, Mauleon R, Li Z. Locating QTLs controlling constitutive root traits in the rice population IAC 165× Co39. Euphytica. 2003;134:335-45.

Darvasi A, Soller M. A simple method to calculate resolving power and confidence interval of QTL map location. Behav Genet. 1997;27(2):125-32.

Dong Y, Kamiunten H, Ogawa T, Tsuzuki E, Terao H, Lin D, et al. Mapping of QTLs for leaf developmental behavior in rice. Euphytica. 2004;138:169-75.

Droc G, Ruiz M, Larmande P, Pereira A, Piffanelli P, Morel JB, et al. OrygenesDB: a database for rice reverse genetics. Nucleic Acids Res. 2006;34:736-40.

Fukai S, Cooper M. Development of drought resistant cultivars using physio-morphological traits in rice. Field Crops Res. 1995;40:6787.

Glaszmann J-C. Isozymes and classification of Asian rice varieties. Theor Appl Genet. 1987;74:21-30.

Goffinet B, Gerber S. Quantitative trait loci: a meta-analysis. Genetics. 2000;155:463-73.

Gomez MS, Kumar SS, Jeyaprakash P, Suresh R, Biji KR, Boopathi $\mathrm{NM}$, et al. Mapping QTLs linked to physio-morphological and plant production traits under drought stress in rice in the target environment. Am J Biochem Biotechnol. 2006;2(4):161-9.

Guo B, Sleper DA, Lu P, Shannon JG, Nguyen HT, Arelli PR. QTLs associated with resistance to soybean cyst nematode in soybean: meta-analysis of QTL locations. Crop Sci. 2006;46:595-602.

Hanocq E, Laperche A, Jaminon O, Lainé AL, Le Gouis J. Most significant genome regions involved in the control of earliness traits in bread wheat, as revealed by QTL meta-analysis. Theor Appl Genet. 2007;114:569-84.

Harushima Y, Yano M, Shomura A, Sato M, Shimano T, Kuboki Y, et al. A high density rice genetic map with 2275 markers using a single $\mathrm{F}_{2}$ population. Genetics. 1998;148:479-94.
Hemamalini GS, Shashidhar HE, Hittalmani S. Molecular marker assisted tagging of morphological and physiological traits under two contrasting moisture regimes at peak vegetative stage in rice. Euphytica. 2000;112:69-78.

Hittalmani S, Huang N, Courtois B, Venuprasad R, Shashidhar HE, Zhuang JY, et al. Identification of QTL for growth- and grain yield-related traits in rice across nine locations of Asia. Theor Appl Genet. 2003;107(4):679-90.

Horii H, Nemoto K, Miyamoto N, Harada J. Quantitative trait loci for adventitious and lateral roots in rice. Plant Breeding. 2006;125:198-200.

Hospital F, Charcosset A. Marker-assisted introgression of quantitative trait loci. Genetics. 1997;147:1469-85.

Hospital F, Goldringer I, Openshaw S. Efficient marker-based recurrent selection for multiple quantitative trait loci. Genet Res. 2000;75:357-68.

International Rice Research Institute (2002) Rice almanac, 253 pp

Kamoshita A, Wade LJ, Ali ML, Pathan MS, Zjang J, Sarkarung S, et al. Mapping QTL for root morphology of a rice population adapted to rainfed lowland conditions. Theor Appl Genet. 2002a;104:880-93.

Kamoshita A, Zhang J, Siopongco J, Sarkarung S, Nguyen HT, Wade L. Effects of phenotyping environment on identification of quantitative trait loci for rice root morphology under anaerobic conditions. Crop Sci. 2002b;42:255-65.

Khowaja F, Norton GJ, Courtois B, Price AH. Improved resolution in the position of drought-related QTLs in a single mapping population of rice by meta-analysis. BMC Genomics 2009; 10:276.

Kojima S, Takahashi Y, Kobayashi Y, Monna L, Sasaki T. Hd3a, a rice ortholog of the Arabidopsis FT gene promotes transition to flowering downstream of Hd1 under short-day conditions. Plant Cell Physiol. 2002;43(10):1096-105.

Lafitte RH, Courtois B. Genetic variation in performance under reproductive-stage water deficit in a doubled-haploid rice population in upland fields. In: Ribaut JM, editor. Molecular approaches for the genetic improvement of cereals for stable production in water-limited environments. Mexico: CIMMYT; 1999. p. 97-102.

Lafitte RH, Champoux MC, McLaren G, O'Toole JC. Rice root morphological traits are related to isozyme groups and adaptation. Field Crops Res. 2001;71:57-70.

Lanceras JC, Pantuwan G, Jongdee B, Toojinda T. Quantitative trait loci associated with drought tolerance at reproductive stage. Plant Physiol. 2004;135:384-99.

Larmande P, Gay C, Lorieux M, Périn C, Bouniol M, Droc G, et al. Oryza Tag Line, a database for the phenotypic characterization of the Génoplante rice insertion line library. Nucleic Acids Res. 2008;36:1022-7.

Li Z, Pinson SRM, Stansel JW, Park WD. Identification of quantitative trait loci for heading date and plant height in cultivated rice. Theor Appl Genet. 1995;91:374-81.

Li RM, Lyons A, Wittenburg H, Paigen B, Churchill GA. Combining data from multiple inbred line crosses improves the power and resolution of QTL mapping. Genetics. 2005a;169:1699-709.

Li Z, Mu P, Li C, Zhang H, Li Z, Gao Y, et al. QTL mapping of root traits in a doubled haploid population from a cross between upland and lowland japonica rice in three environments. Theor Appl Genet. 2005b;110:1244-52.

Lin HX, Yamamoto T, Sasaki T, Yano M. Characterization and detection of epistatic interactions of 3 QTLs, Hd1, Hd2, and $\mathrm{Hd} 3$, controlling heading date in rice using nearly isogenic lines. Theor Appl Genet. 2000;101:1021-8.

Ludlow MM, Muchow RC. A critical evaluation of traits for improving crop yield in water-limited environments. Adv Agron. 1990;43:107-53. 
MacMillan K, Emrich K, Piepho H-P, Mullins CE, Price AH. Assessing the importance of genotype $\mathrm{x}$ environmental interaction for root traits in rice using a mapping population II: conventional QTL analysis. Theor Appl Genet. 2006;113:95364.

Maheswaran M, Huang N, Sreerangasamy SR, McCouch SR. Mapping quantitative trait loci associated with days to flowering and photoperiod sensitivity in rice. Mol Breed. 2000;6:145-55.

Malamy JE. Intrinsic and environmental response pathways that regulate root system architecture. Plant Cell Environ. 2005;28:67-77.

Mather KA, Caicedo AL, Polat NR, Olsen KM, McCouch S, Purruganan MD. The extent of linkage disequilibrium in rice. Genetics. 2007;177:2223-32.

Monna L, Kitazawa N, Yoshino R, Suzuki J, Masuda H, Mahera Y, et al. Positional cloning of rice semi-dwarfism gene $s d-1$ : rice "green revolution gene" encodes a mutant enzyme involved in gibberellin synthesis. DNA Res. 2000;9(1):11-7.

Nemoto K, Morita S, Baba T. Shoot and root development in rice related to the phyllochron. Crop Sci. 1995;35:24-9.

Nguyen HT, Babu RC, Blum A. Breeding for drought resistance in rice: physiology and molecular considerations. Crop Sci. 1997;37:1426-34.

Nguyen TTT, Klueva N, Chamareck V, Aarti A, Magpantay G, Millena ACM, et al. Saturation mapping of QTL regions and identification of putative candidate genes for drought tolerance. Mol Gen Genomics. 2004;272:35-46.

Nibau C, Gibbs DJ, Coates JC. Branching out in new directions: the control of root architecture by lateral root formation. New Phytol. 2008;179(3):595-614.

Norton GJ, Aitkenhead MJ, Khowaja FS, Whalley WR, Price AH. A bioinformatic and transcriptomic approach to identifying positional candidate genes without fine mapping; an example using rice root-growth QTLs. Genomics. 2008;92:344-52.

O'Toole JC, Bland WL. Genotypic variation in crop plant root systems. Adv Agron. 1987;41:91-145.

Price AH, Steele KA, Townend J, Gorham J, Audebert A, Jones M, et al. Mapping root and shoot and root traits in rice: experience in UK, IRRI and WARDA. In: Ito O, O'Toole J, Hardy B, editors. Genetic improvement of rice for water-limited environments. Manila: IRRI; 1999. p. 257-73.

Price AH, Steele KA, Moore BJ, Barraclough PB, Clark LJ. A combined RFLP and AFLP linkage map of upland rice used to identify QTLs for root-penetration ability. Theor Appl Genet. 2000;100:49-56.

Price AH, Steele KA, Moore BJ, Jones RGW. Upland rice grown in soil filled chambers and exposed to contrasting water-deficit regimes. II. Mapping QTLs for root morphology and distribution. Field Crop Research. 2002;76:25-43.

Ray JD, Yu L, McCouch SR, Champoux MC, Wang G, Nguyen HT. Mapping quantitative trait loci associated with root penetration ability in rice. Theor Appl Genet. 1996;92:627-36.

Rebouillat J, Dievart A, Verdeil JL, Escoute J, Giese E, Breitler JC, et al. Molecular genetics of rice root development. Rice. 2008;2(1):15-34.

Redona ED, Mackill DJ. Mapping quantitative traits loci for seedling vigor in rice using RFLPs. Theor Appl Genet. 1996;92:395-402.

Ruiz M, Rouard M, Raboin LM, Lartaud M, Lagoda P, Courtois B. TropGENE-DB, a multi tropical crop information system. Nucleic Acids Res. 2004;32:364-7.

Servin B, Martin OC, Mezard M, Hospital F. Toward a theory of marker-assisted gene pyramiding. Genetics. 2004;168:513-23.

Shen L, Courtois B, McNally KL, Robin S, Li Z. Evaluation of nearisogenic lines of rice introgressed with QTLs for root depth through marker-aided selection. Theor Appl Genet. 2001;103(1):75-83.
Steele KA, Price AH, Sashidhar HE, Witcombe JR. Marker-assisted selection to introgress rice QTLs controlling root traits into an Indian upland rice variety. Theor Appl Genet. 2006;112:208-21.

Takahashi Y, Shomura A, Sasaki T, Yano M. Hd6, a rice quantitative trait locus involved in photoperiod sensitivity, encodes the $\alpha$ subunit of protein kinase CK2. Proc Natl Acad Sci. 2001;98:7922-7.

Tripathy JN, Zhang J, Robin S, Nguyen TT, Nguyen HT. QTLs for cell-membrane stability mapped in rice under drought stress. Theor Appl Genet. 2000;100:1197-202.

Veyrieras JB, Goffinet B, Charcosset A. Meta QTL: a package of new computational methods for the meta-analysis of QTL mapping experiments. BMC Bioinformatics. 2007;8:49.

Widawski DA, O'Toole JC. Prioritizing the rice biotechnology research agenda for eastern India. New York: The Rockefeller Foundation; 1990. 86.

Xu CG, Li XQ, Xue Y, Huang YW, Gao J, Xing YZ. Comparison of quantitative trait loci controlling seedling characteristics at two seedling stages using rice recombinant inbred lines. Theor Appl Genet. 2004;109:640-7.

Yadav R, Courtois B, Huang N, McLaren G. Mapping genes controlling root morphology and root distribution in a doubledhaploid population of rice. Theor Appl Genet. 1997;94:619-32.

Yamamoto T, Lin H, Sasaki T, Yano M. Identification of heading date quantitative trait locus Hd6 and characterization of its epistatic interactions with $\mathrm{Hd} 2$ in rice using advanced backcross progeny. Genetics. 2000;154:885-91.

Yambao EB, Ingram KT, Real JG. Root xylem influence on the water relations and drought resistance in rice. J Exp Bot. 1992;43: 925-32.

Yano M, Harushima Y, Nagamura Y, Kurata N, Minobe Y, Sasaki T. Identification of quantitative trait loci controlling heading date in rice using a high-density linkage map. Theor Appl Genet. 1997;95:1025-32.

Yano M, Katayose Y, Ashikari M, Yamanouchi U, Monna L, Fuse T, et al. $H d l$, a major photoperiod sensitivity quantitative trait locus in rice, is closely related to the Arabidopsis flowering time gene CONSTANS. Plant Cell. 2000;12:2473-84.

Yoshida S, Hasegawa S. The rice root system: its development and function. In: O'Toole JC, editor. Drought resistance in crops with emphasis on rice. Los Baños, Laguna: IRRI; 1982. p. 97-114.

Yu SB, Li JX, Xu CG, Tan YF, Li XH, Zhang Q. Identification of quantitative trait loci and epistatic interactions for plant height and heading date in rice. Theor Appl Genet. 2002;104:619-25.

Yue B, Xue W, Xiong L, Yu X, Luo L, Cui K, et al. Genetic basis of drought resistance at reproductive stage in rice: separation of drought tolerance from drought avoidance. Genetics. 2006; 172:1213-28.

Zhang J, Zheng HG, Aarti A, Pantuwan G, Nguyen TT, Tripathy JN, et al. Locating genomic regions associated with components of drought resistance in rice: comparative mapping within and across species. Theor Appl Genet. 2001a;103:19-29.

Zhang WP, Shen XY, Wu P, Hu B, Liao CY. QTL and epistasis for seminal root length under a different water supply in rice. Theor Appl Genet. 2001b;103:118-23.

Zheng HG, Babu RC, Pathan MS, Ali L, Huang N, Courtois B, et al. Quantitative trait loci for root-penetration ability and root thickness in rice: comparison of genetic backgrounds. Genome. 2000;43:53-61.

Zheng BS, Yang L, Zhang WP, Mao CZ, Wu YR, Yi KK, et al. Mapping QTLs and candidate genes for rice root traits under different water-supply conditions and comparative analysis across three populations. Theor Appl Genet. 2003;107:1505-15. 